\title{
Studies on dual readout calorimetry with mela-crystals
}

Etiennette Auffray, Paul Lecoq, Georgios Mavromanolakis

European Organization for Nuclear Research CERN, CH-1211 Geneva, Switzerland

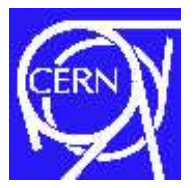

Outline

- Introduction - General

- Calorimetry with meta-crystals

- Dual readout and energy correction

- Case studies and simulation results

- Questions - Outlook 


\section{Introduction - General}

-. $\mathbf{R} \& \mathbf{D}$ for future hep calorimetry : mainly 3 lines of approach

$\triangleright$. particle flow approach (CALICE)

$\triangleright$. dual readout calorimetry (DREAM)

$\triangleright$. crystal calorimetry (e.g. see HHCAL workshop)

$\triangleright$. particle flow paradigm

highly granular EM and HADR calorimeters to allow very efficient pattern recognition for excellent shower separation and pid within jets to provide excellent jet reconstruction efficiency

\section{$\triangleright$. dual readout calorimetry}

measurement of both the ionisation/scintillation and the Cherenkov signals generated by a hadronic shower in order to determine on an event by event basis the electromagnetic fraction of the shower and so to cancel/correct for this source of fluctuation that degrades the energy resolution of the calorimeter

\section{. crystal calorimetry}

an approach that could combine the excellent energy resolution of crystals (homogeneous detector) with dual readout, if scintillation and Cherenkov signals can be separated and recorded, and with particle flow/imaging capabilities if the detector is segmented with high granularity 


\section{Dual readout with metamaterials}

\section{- the meta-crystals concept}

: use of both undoped and $\mathrm{Ce}$ doped heavy crystal fibers of identical material. The undoped crystals behave as Cherenkov radiators while the doped crystals behave as scintillators

: a candidate material is the Lutetium Aluminium Garnet (LuAG) crystal

$$
\left(\mathrm{Lu}_{3} \mathrm{Al}_{5} \mathrm{O}_{12}\right)
$$

\begin{tabular}{llll}
\hline \hline Physical properties & \multicolumn{3}{l}{ Optical properties } \\
\hline Density & $6.73 \mathrm{gr} / \mathrm{cm}^{3}$ & Light yield (Ce doped) & $25000 \mathrm{ph} / \mathrm{MeV}$ (50\% of Nal) \\
Zeff & 62.9 & Emission wavelength & $535 \mathrm{~nm}$ (Ce doped) \\
Radiation length $X_{0}$ & $1.41 \mathrm{~cm}$ & Decay time & $60 \mathrm{nsec}($ Ce doped) \\
Interaction length $\lambda_{I}$ & $23.3 \mathrm{~cm}$ & Refractive index & 1.842 at $633 \mathrm{~nm}$ \\
Melting point & $2260{ }^{\circ} \mathrm{C}$ & Cherenkov threshold & $97 \mathrm{keV}$ \\
Thermal expansion & $8.810^{-6} /{ }^{\circ} \mathrm{C}$ & Max Cherenkov angle & $57^{\circ}$ \\
\hline Thermal conductivity & $31 \mathrm{~W} / \mathrm{m}^{\circ} \mathrm{C}$ & Total reflection angle & $33^{\circ}$ \\
\hline \hline
\end{tabular}




\section{$\mathbf{R} \& \mathbf{D}$ activities}

- material development

: comprehensive program of studies within the framework of the Crystal Clear Coll. with focus on hep and medical imaging applications

- testbeam activities

: very first testbeam studies with bundles of fibers exposed to electron beam

: small scale tests i.e. equivalent to a level of a single calorimetric channel

: data collection during Sep08, May09, Nov09

- simulation studies

: systematic scanning of the parametric space wrt granularity, sampling fraction, readout fraction, total length etc, for first understanding of performance trends and showstoppers and to proceed from an ideal case to a realistic one 


\section{Crystal fiber production}

\section{Micro-pulling down method}

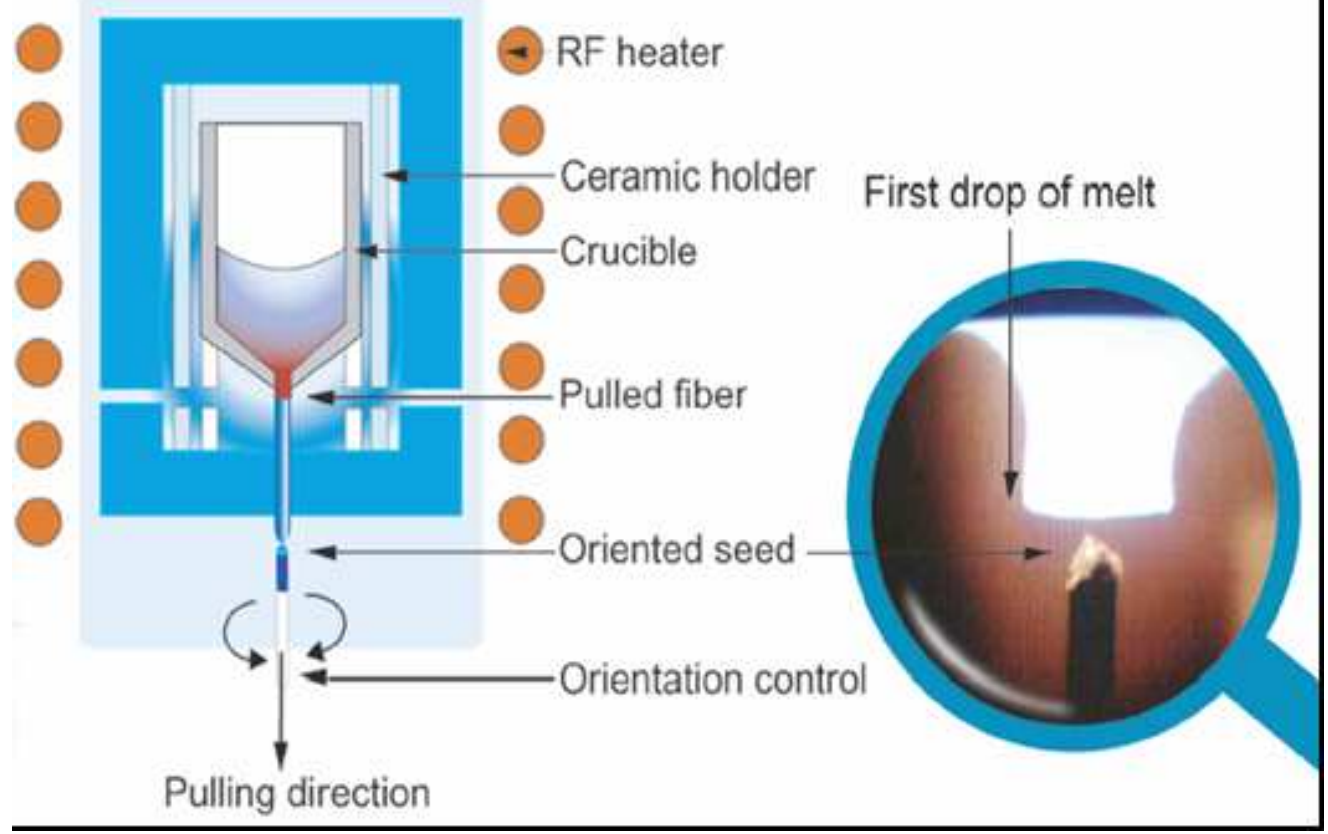

(courtesy of Fibercryst-Lyon, Cyberstar-Grenoble)

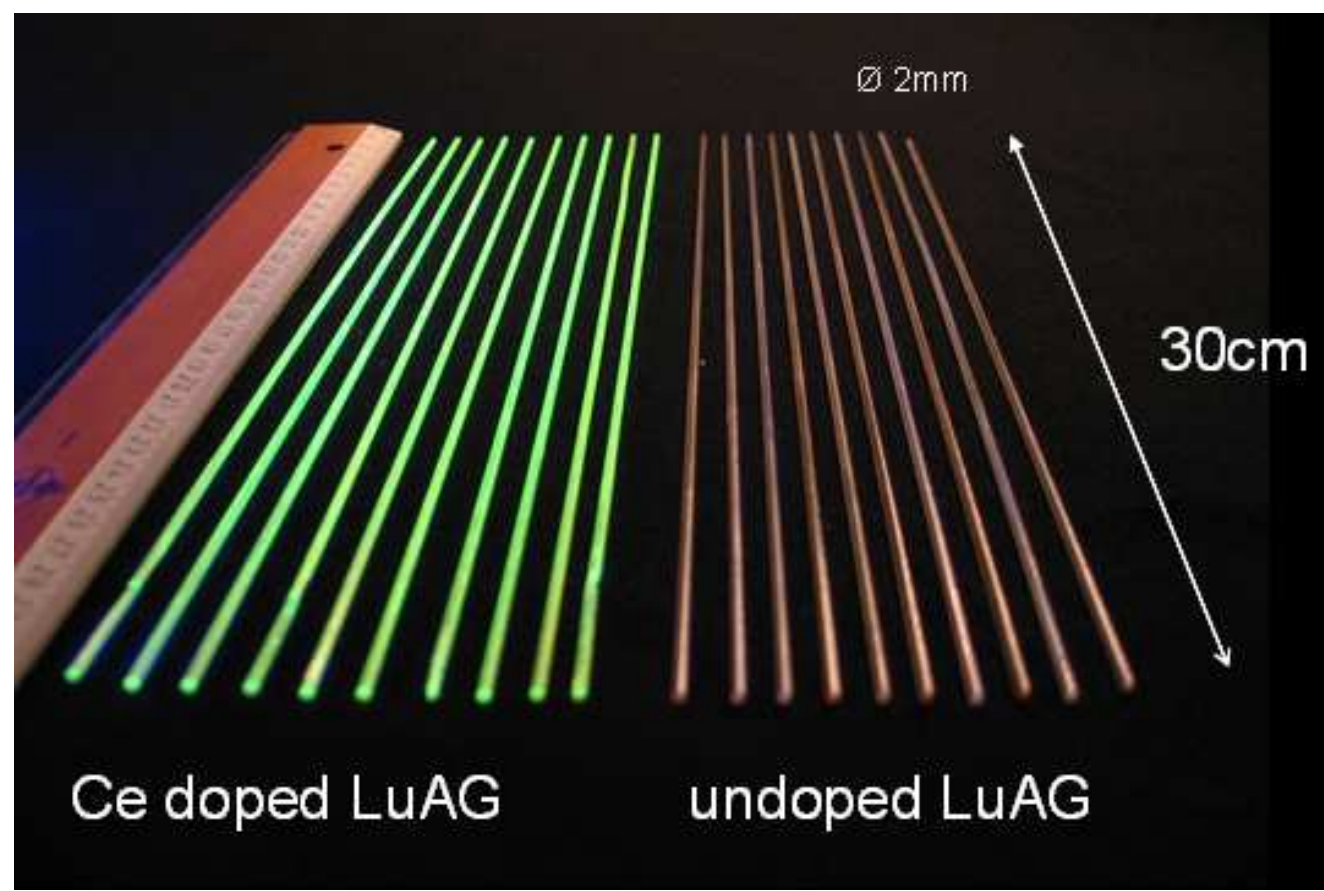

(20 fibers of diameter $=2 \mathrm{~mm}$, length $=30 \mathrm{~cm}$ )

- fiber diameter between 0.3-3 mm, length up to $2 \mathrm{~m}$

- pulling rate ranging from 0.1 to $0.5 \mathrm{~mm} / \mathrm{min}$

- capillary die can be non-cylindrical (e.g. square, hexagonal etc)

- overall cost per unit volume of production expected to be comparable to that of standard crystal growth methods 


\section{Concept of a readout unit}

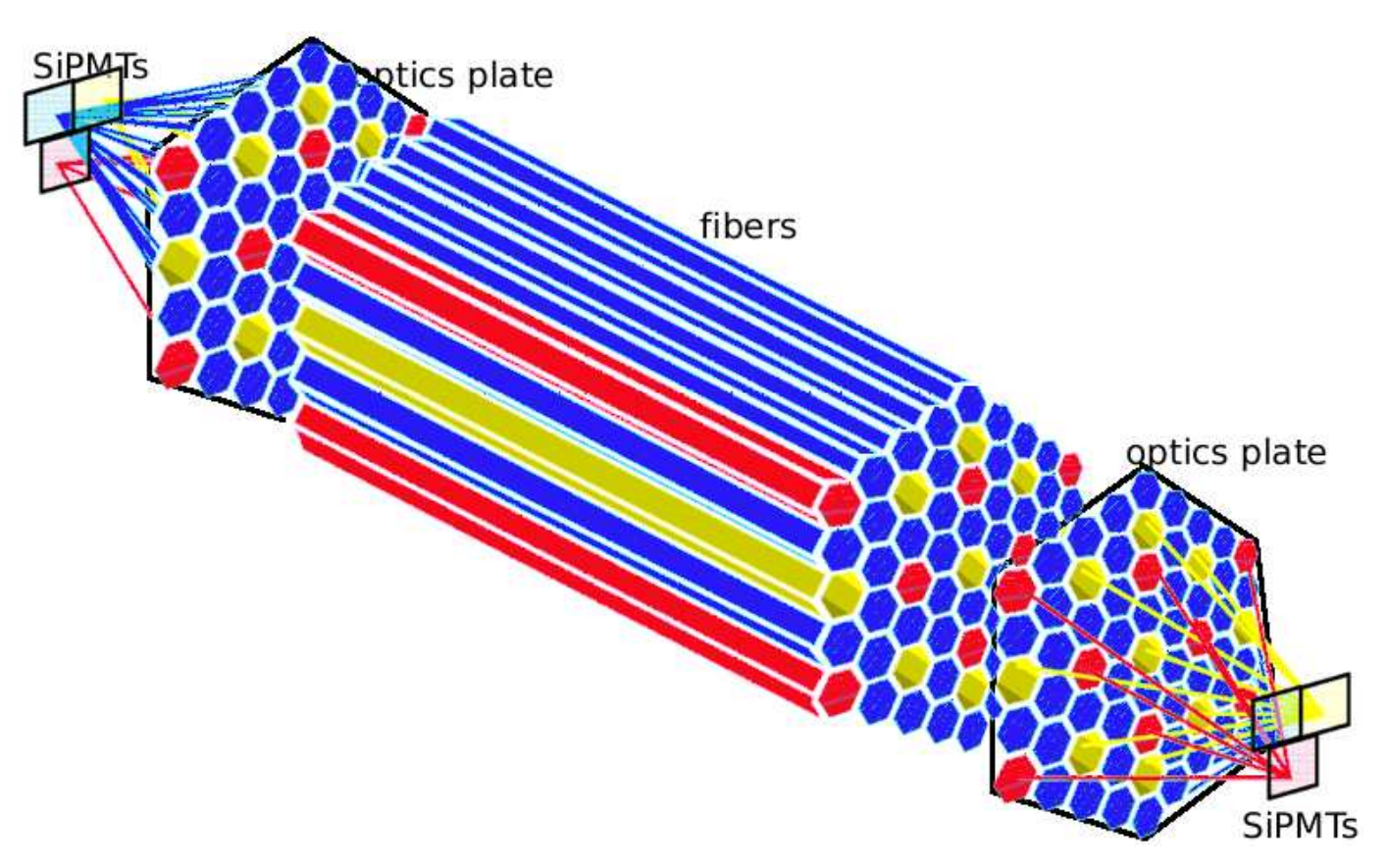

- a unit consists of a structured distribution of different types of fibers

- typical dimensions of a unit : $\mathrm{d}=1-1.5 \mathrm{R}_{\mathrm{M}}, \mathrm{L}=20-25 \mathrm{X}_{0}$

- light from different types of fibers is directed to different SiPMTs by using diffractive optics light concentrators (micro-lenses)

- diffractive optics plate with pattern to match the structure of fibers 


\section{Crystal fiber studies - material development}
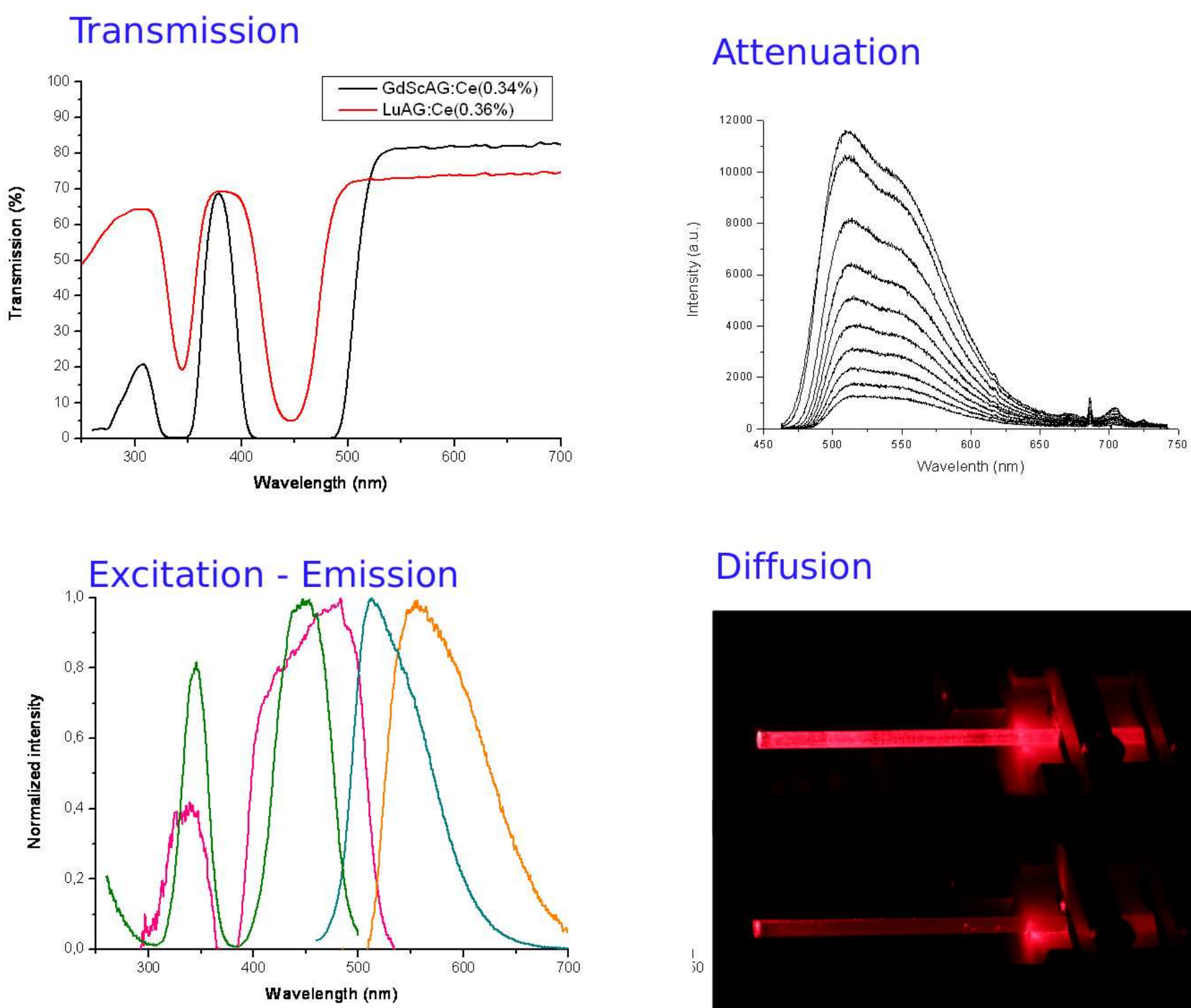

\section{Diffusion}

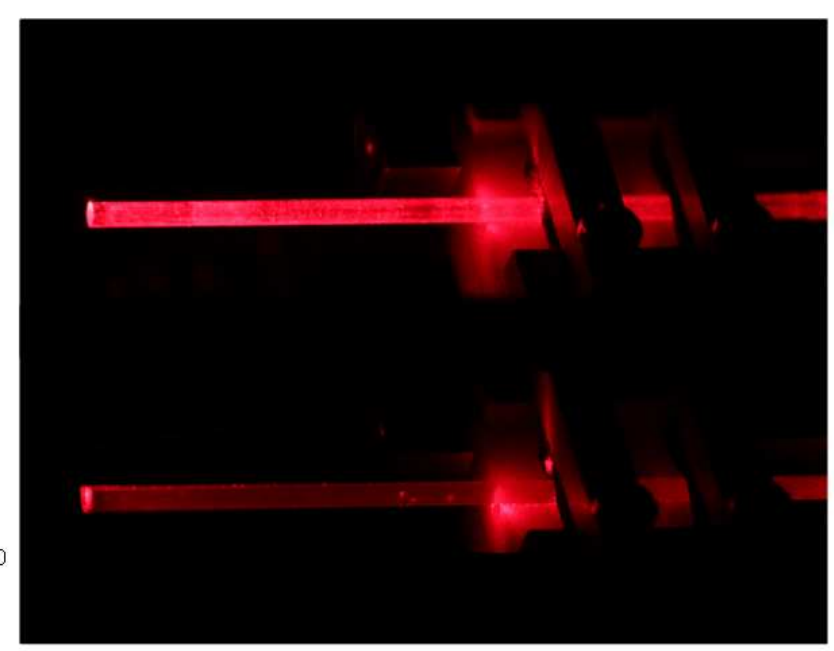

Exclation spectrum GaScAG.Ce(0.34\%)

Em ission spectrum GdSCAG:Ce(0.34\%)

Excitation spectrum LuAG:Ce( $(0.36 \%)$ 


\section{Fiber bundles exposed to beam}

Ce doped LuAG scintillator

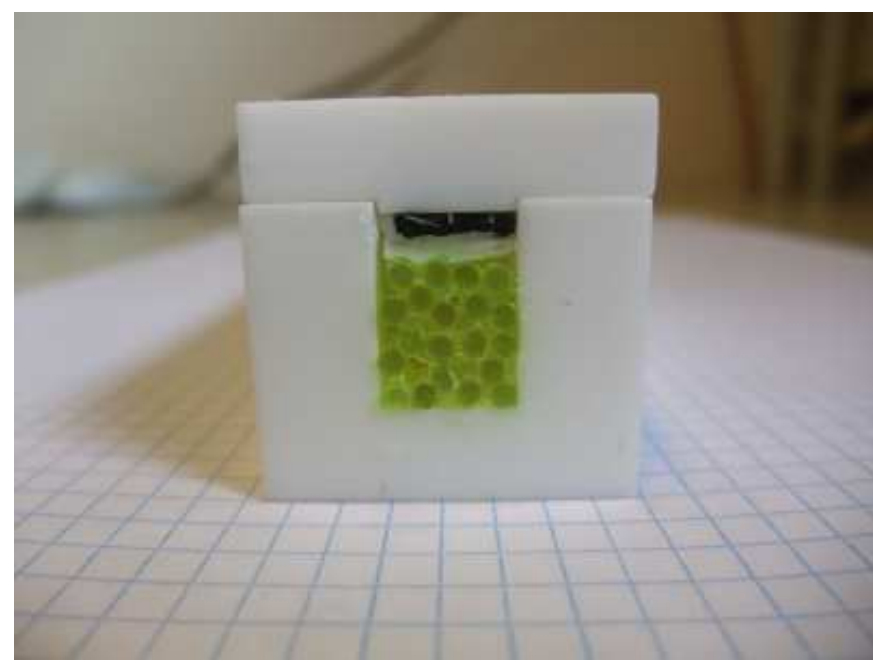

(20 fibers of diameter $=2 \mathrm{~mm}$, length $=80 \mathrm{~mm}$ )

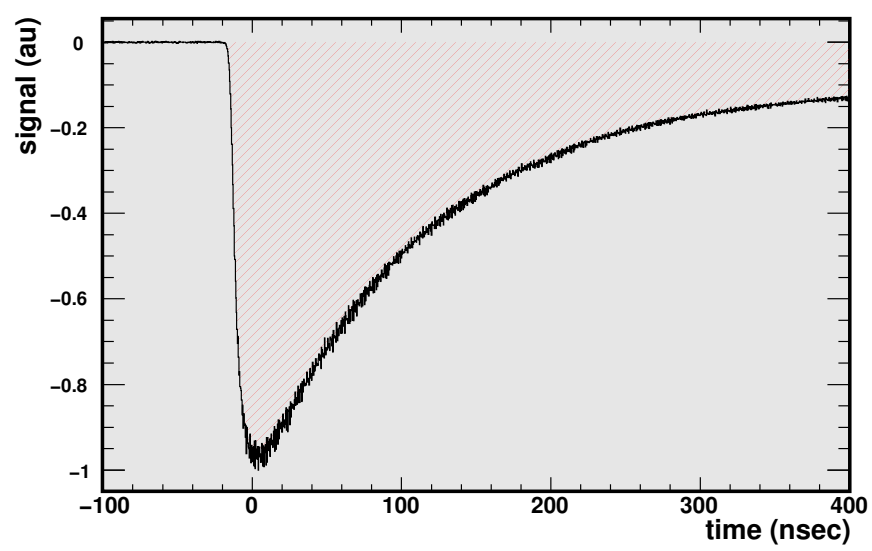

undoped LuAG

Cherenkov radiator

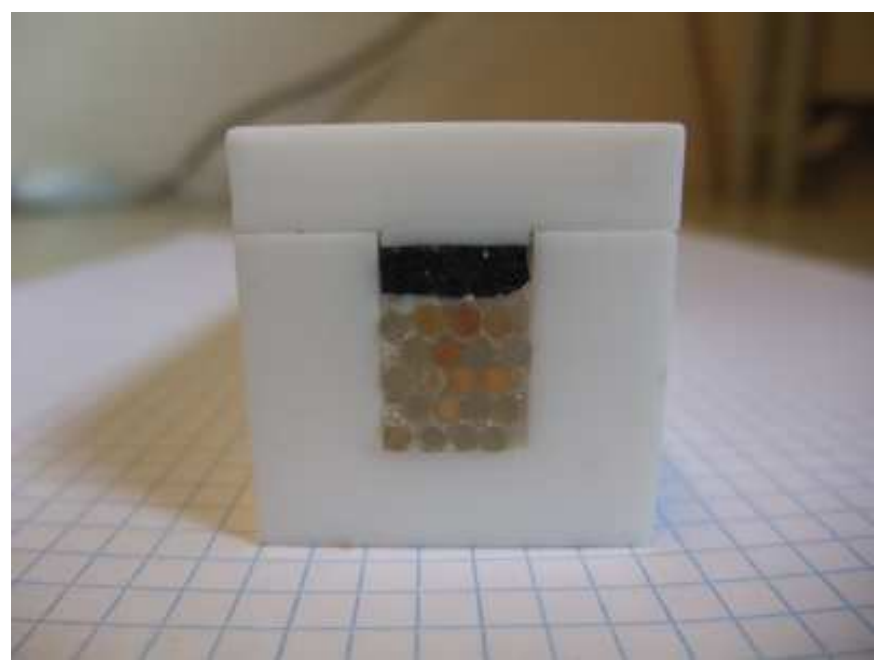

(20 fibers of diameter $=2 \mathrm{~mm}$, length $=80 \mathrm{~mm}$ )

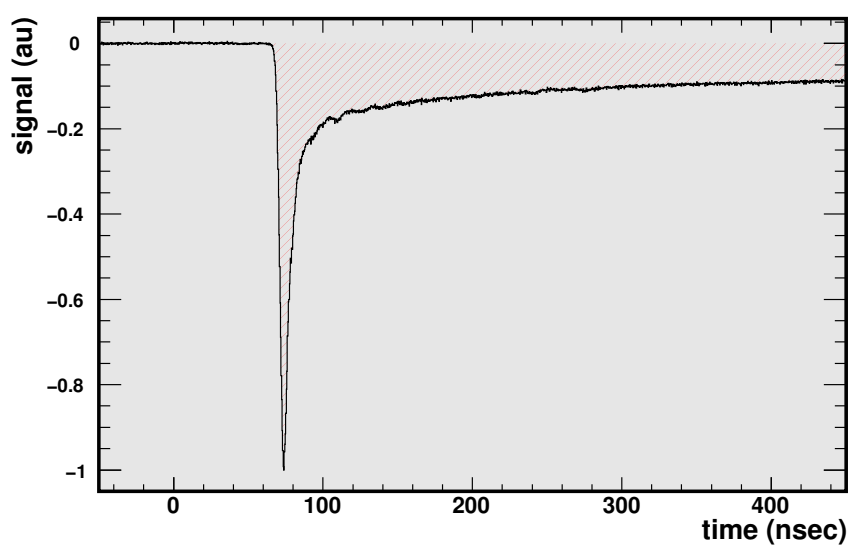




\section{Testbeam results}
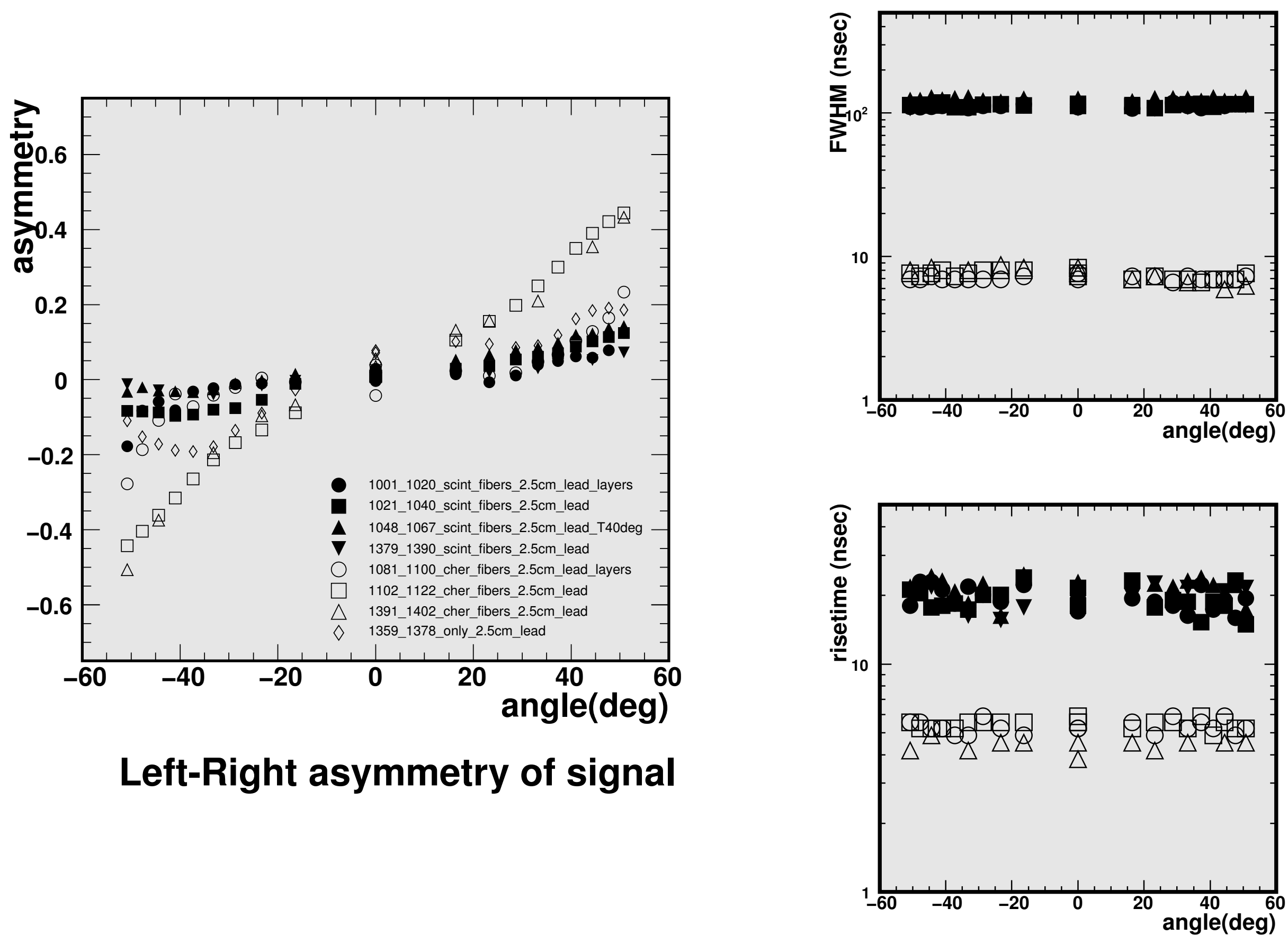


\section{Dual-readout method}

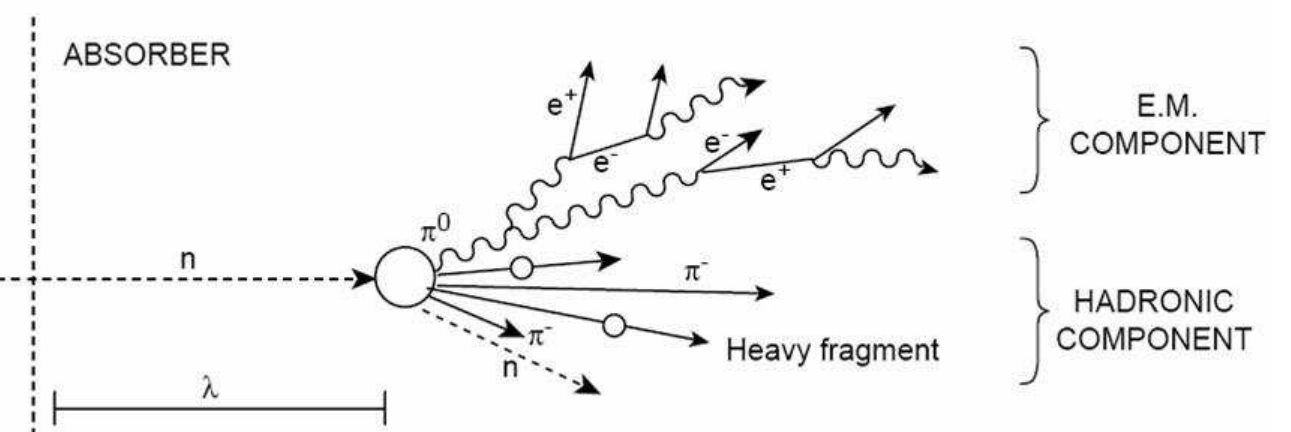

JV215.c

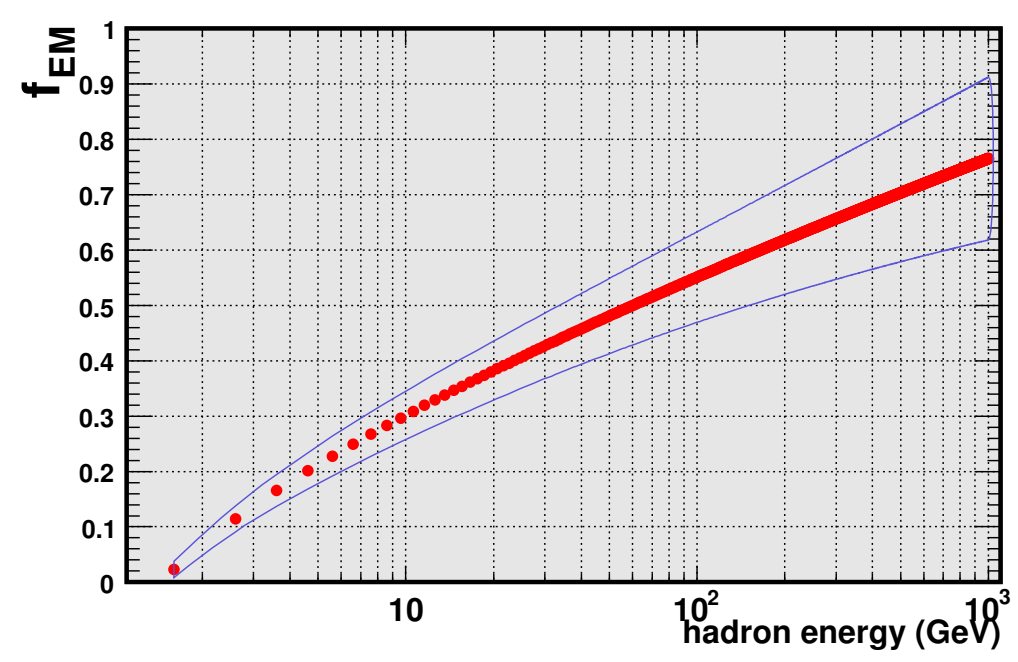

a hadron shower develops an electromagnetic (through $\pi^{0} \rightarrow \gamma \gamma$ ) and a hadronic component with relative ratio $f_{E M}$. The ratio fluctuates on event-by-event and degrades the energy resolution of a hadronic calorimeter.

- With the dual-readout technique one aims to measure and so to correct/cancel this source of fluctuation on an event-by-event basis. This is done by recording simultaneously the signals generated by two different signal generation mechanisms.

- signal 1: $S=\epsilon_{S} \cdot f_{E M} \cdot E+h_{S} \cdot\left(1-f_{E M}\right) \cdot E$ signal 2: $Q=\epsilon_{Q} \cdot f_{E M} \cdot E+h_{Q} \cdot\left(1-f_{E M}\right) \cdot E$ hence $\mathrm{S} / \mathrm{E}=\mathrm{c} /(1-\mathrm{k} \cdot \mathrm{Q} / \mathrm{S})=F(\mathrm{Q} / \mathrm{S})$ 


\section{Dual readout and energy correction}

\section{- correct Eionz for single pions}

: define CorrectionFactor $=1$ - calibr ${ }^{*}$ Echer/Eionz

with calibr $=$ Eionz/Echer for electrons at given energy

: get correction function Fionz() by fitting Eionz vs CorrectionFactor of single pions at given energy

: corrected energy $=$ Eionz/Fionz(), applied to pions of various energies

Or equivalently

\section{- correct Echer for single pions}

: define CorrectionFactor $=1$ - calibr * Echer/Eionz with calibr $=$ Eionz/Echer for electrons at given energy

: get correction function Fcher() by fitting Echer vs CorrectionFactor of single pions at given energy

: corrected energy $=$ Echer/Fcher(), applied to pions of various energies
hEion_VS_CorrectionFactor

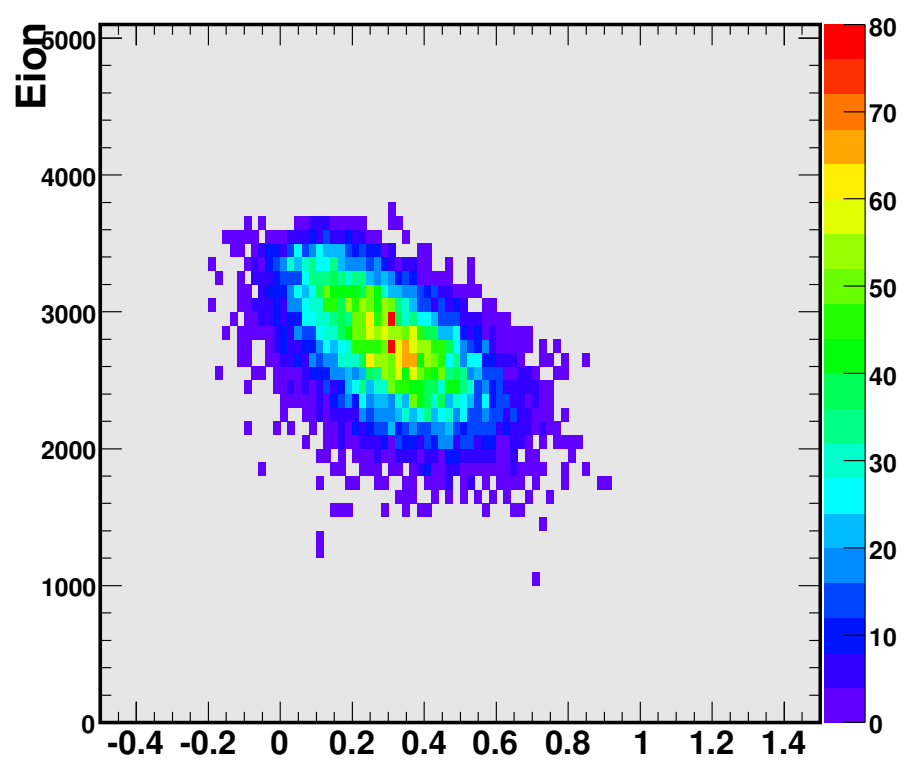

hEcher_VS_CorrectionFactor

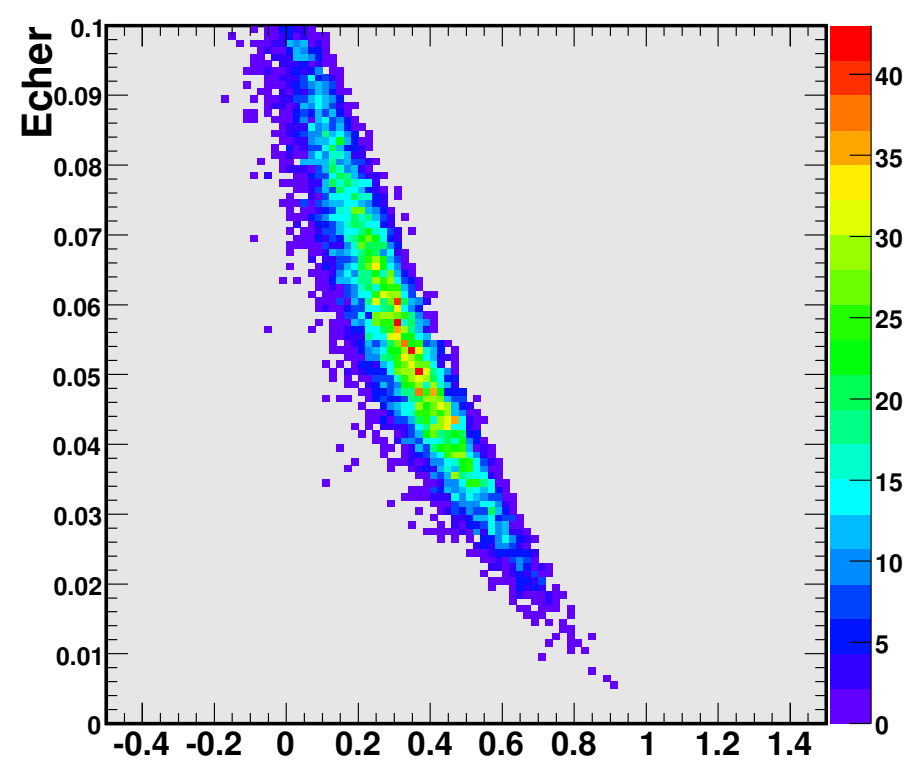




\section{case of an homogeneous detector}

$\pi^{-1} \mathrm{GeV}$
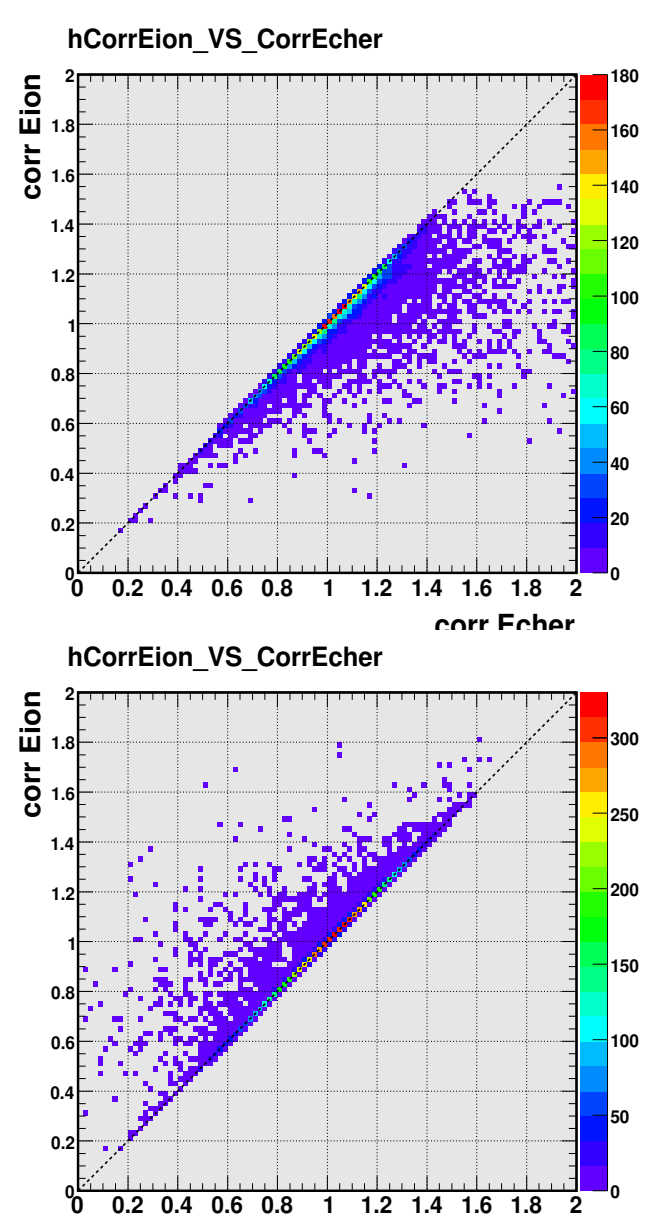

enrr Frher

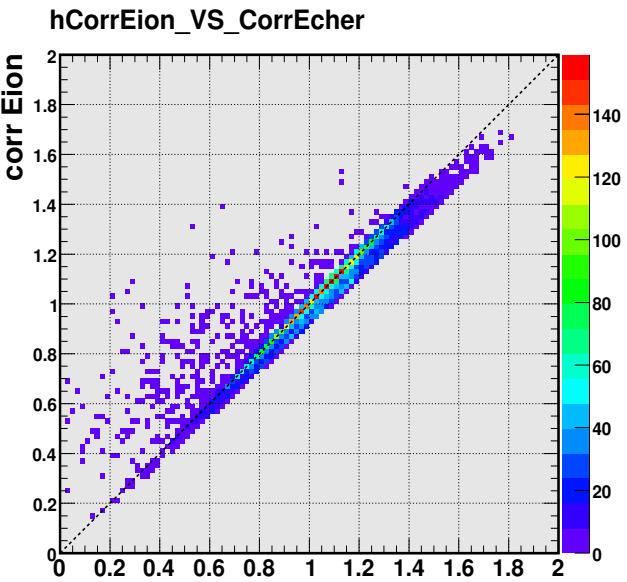

$\begin{array}{ccccccccccc}0 & 0.2 & 0.4 & 0.6 & 0.8 & 1 & 1.2 & 1.4 & 1.6 & 1.8 & 2\end{array}$ $\pi^{-} 5 \mathrm{GeV}$

hCorrEion_vs_CorrEcher

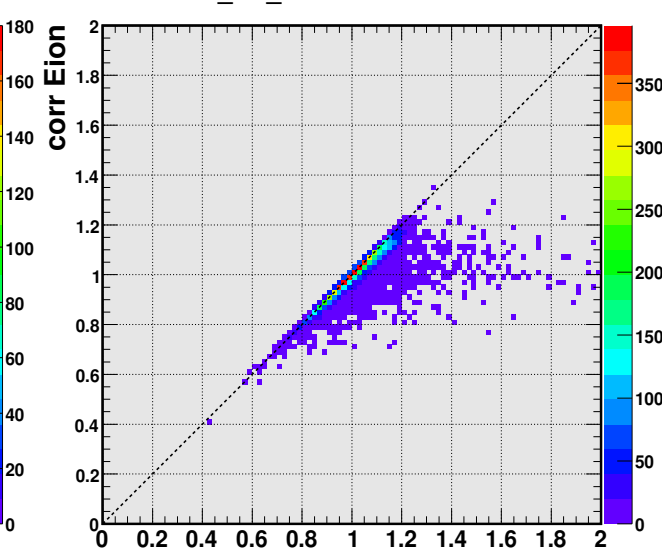

norr Frhar

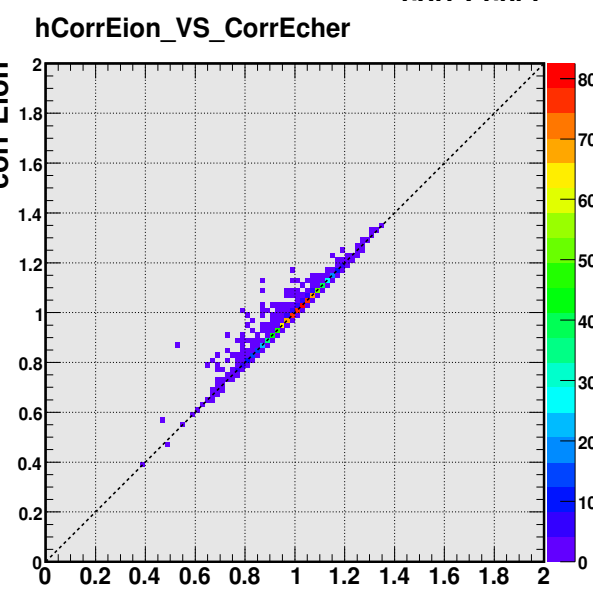

hCorrEion_VS_CorrEcher

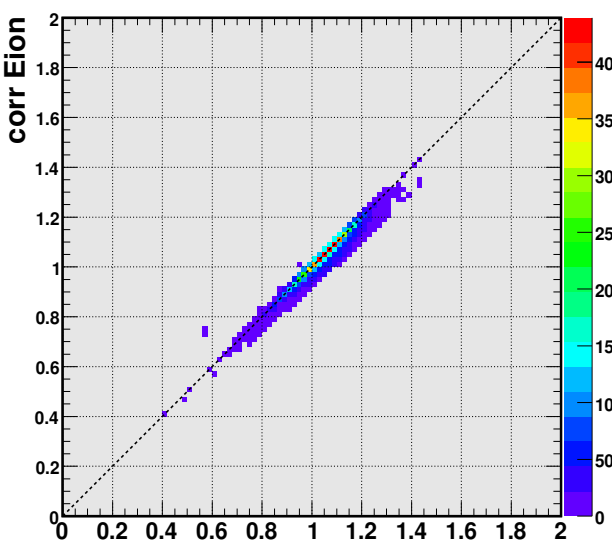

12. G.Mavromanolakis, 101020, IWLC2010

$\pi^{-10} \mathbf{G e V}$

hCorrEion_vs_CorrEcher

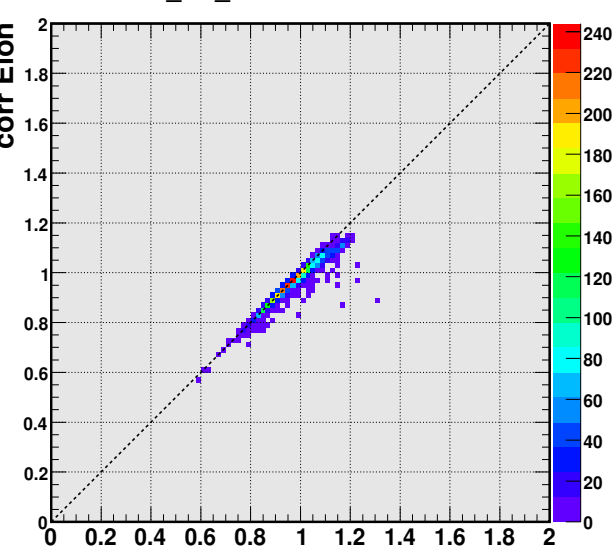

enrr Frhar

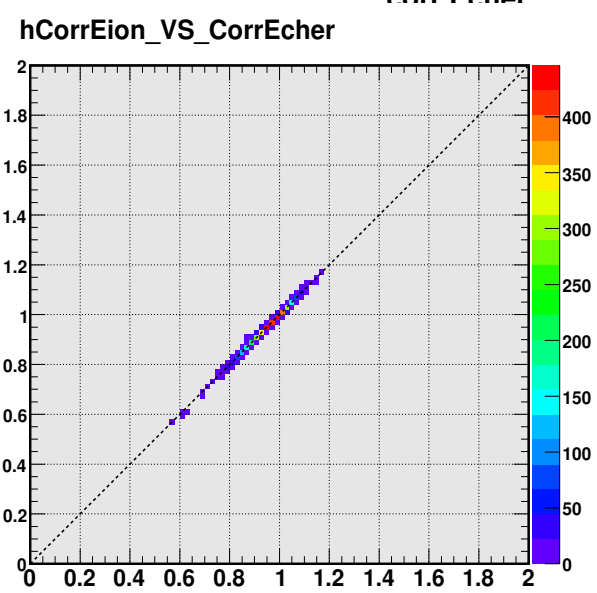

corr Frher

hCorrEion_VS_CorrEcher

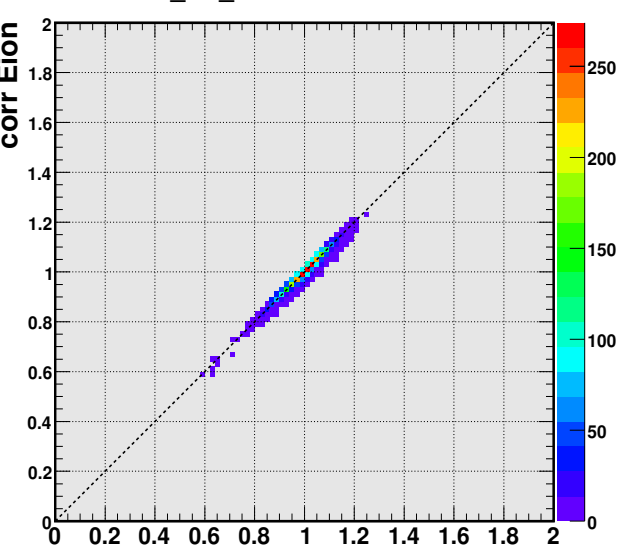




\section{Case studies}

- in brief

: systematic scanning of the parametric space with respect to

- granularity

- sampling fraction

- readout fraction

- total length

- mixture of conventional and dual readout components

- mixture of homogeneous and sampling components

- corresponding composition, etc

$\checkmark$. in the following

: discuss an ideal calorimeter without leakage $\left(4.3 \times 4.3 \times 8.6 \lambda_{l}^{3}\right)$ (corresponding to a $1 \times 1 \times 2 \mathrm{~m}^{3}$ volume composed of LuAG) 


\section{Correlation plots for various readout fractions}

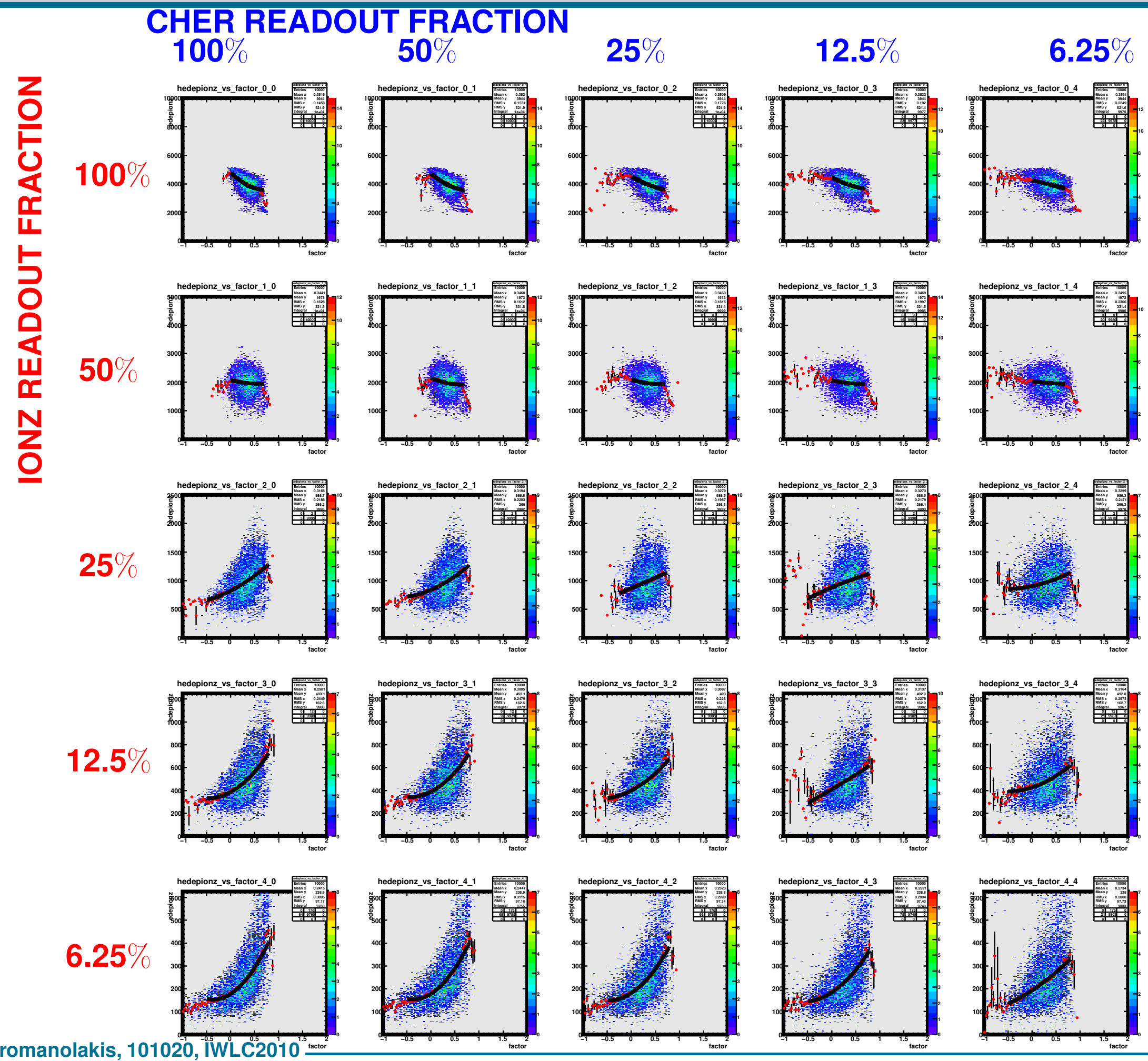




\section{Energy resolution vs readout fraction}
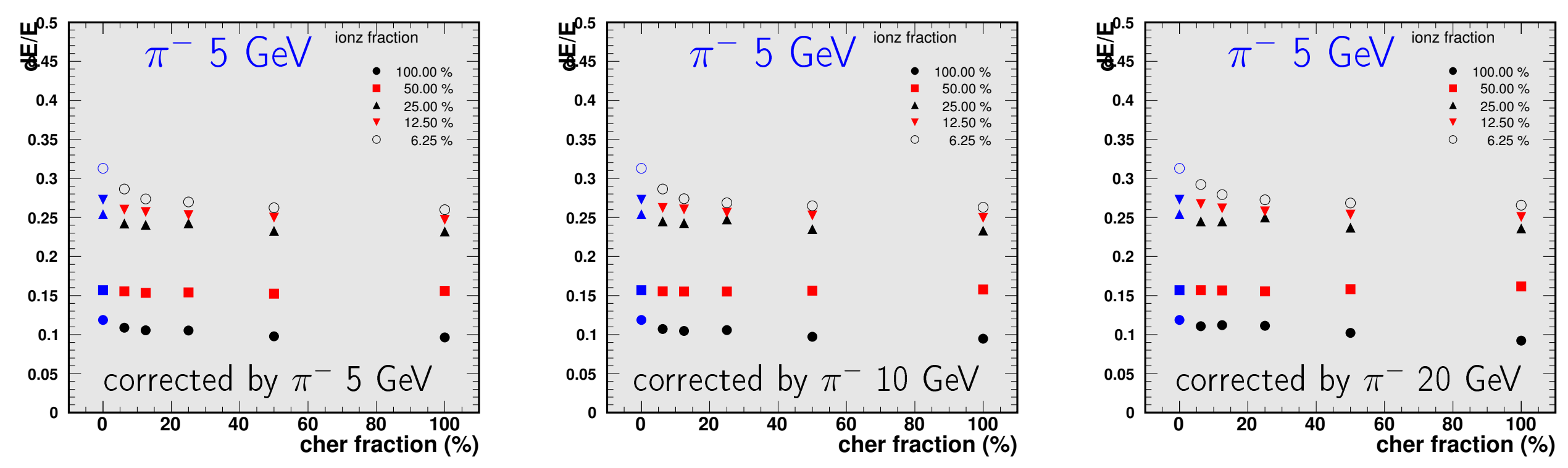

similar results with different correction energies
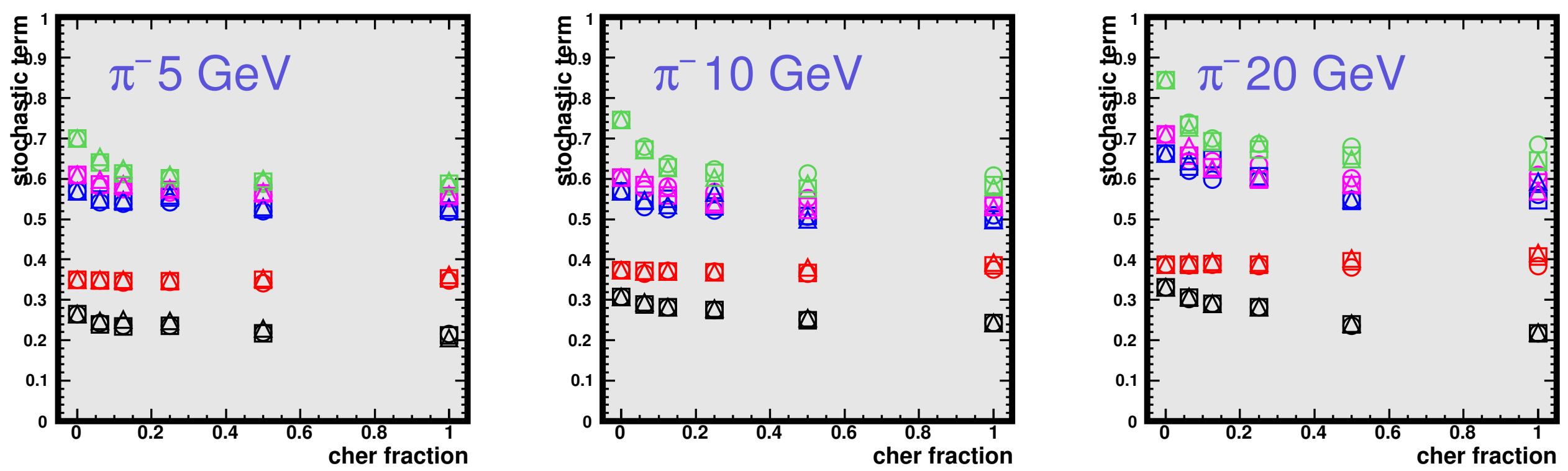

(different colors denote different ionz readout fractions)

(different symbols denote different energy samples used for correction) 


\section{Stochastic term vs readout fraction and length}

case of a calorimeter of $\approx 4.3 \times 4.3 \times 8.6 \lambda_{\mathrm{I}}^{3}$

$\left(1 \times 1 \times 2 \mathrm{~m}^{3}\right.$ LuAG)

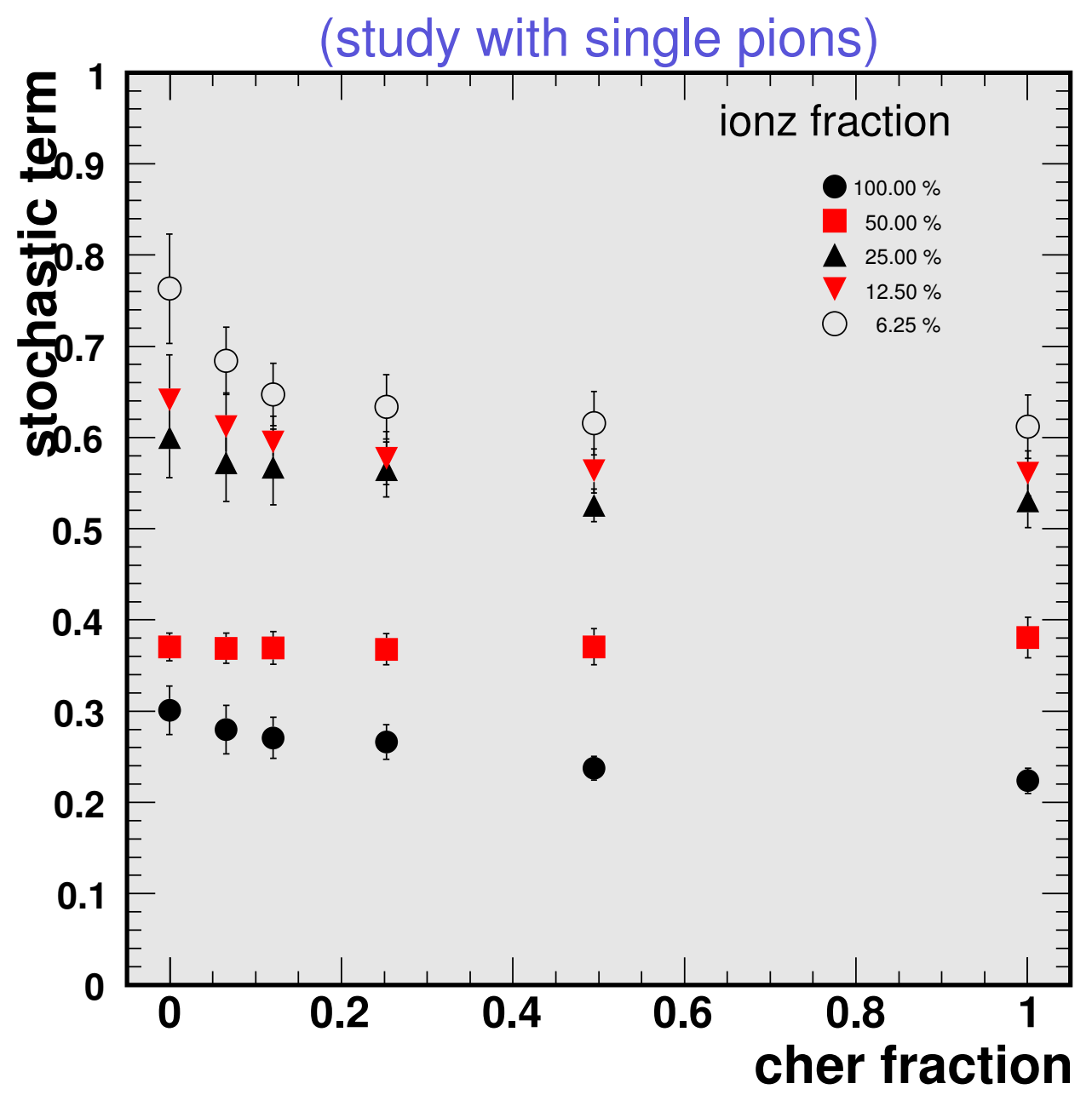

(change of readout fractions at full depth)
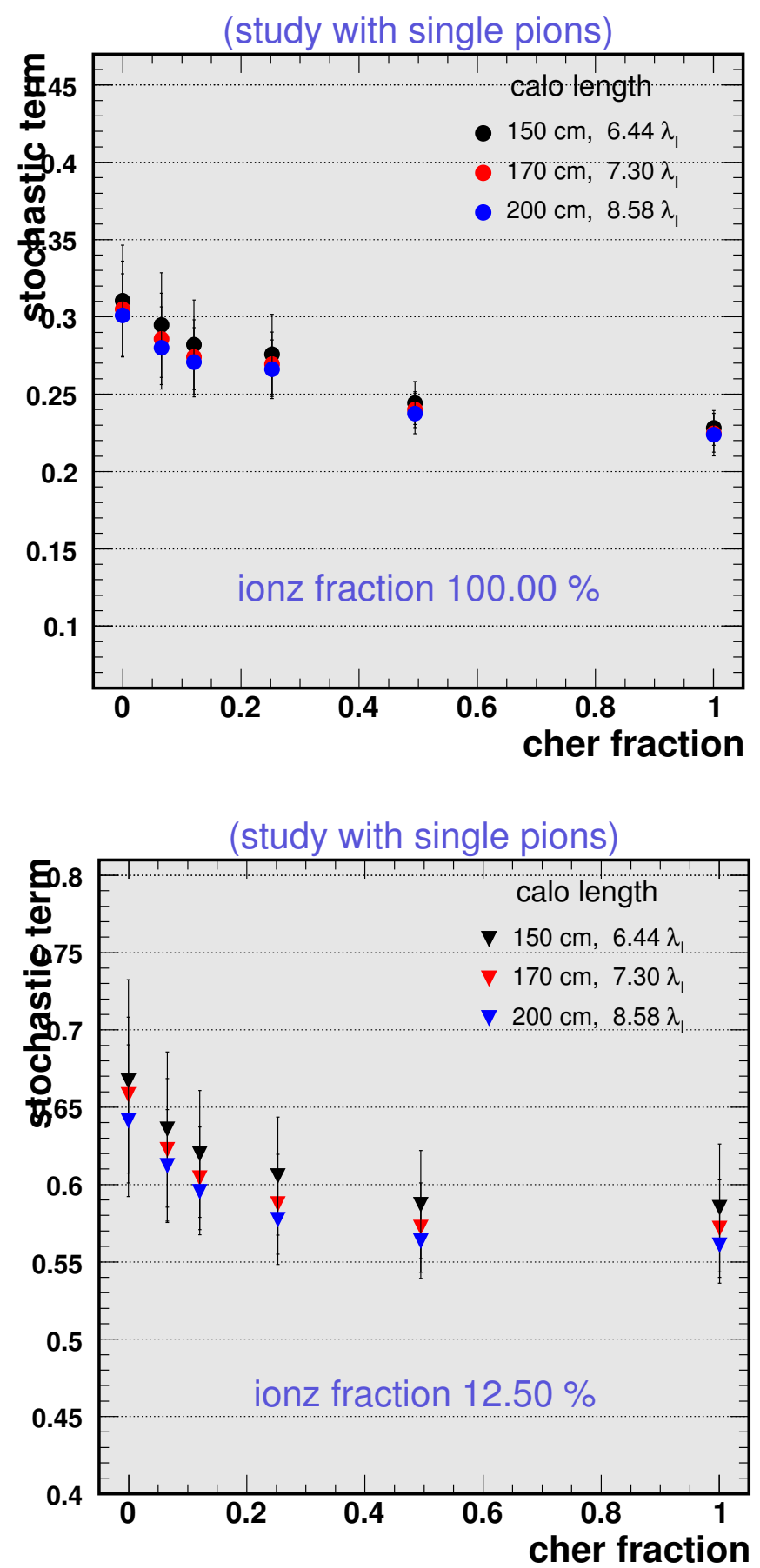


\section{Stochastic term for various configurations}

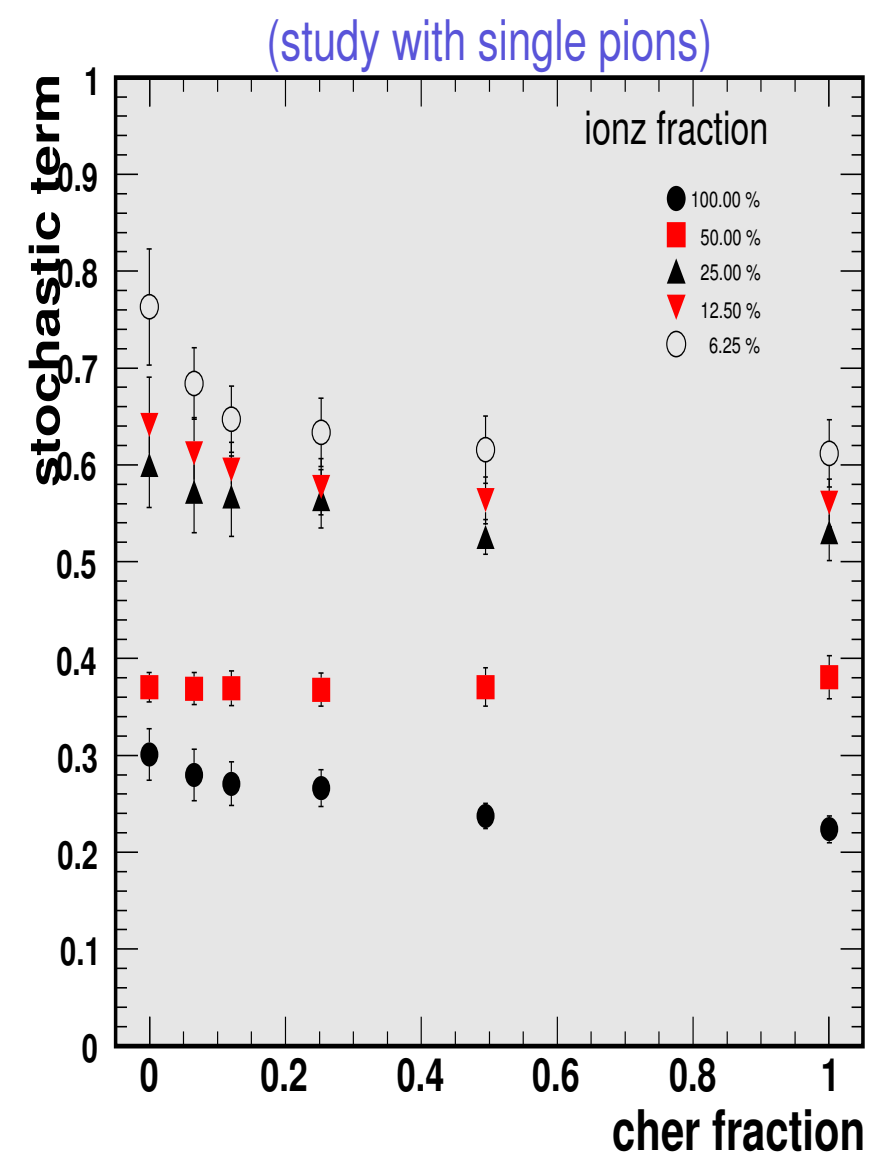

change of readout fractions at full depth

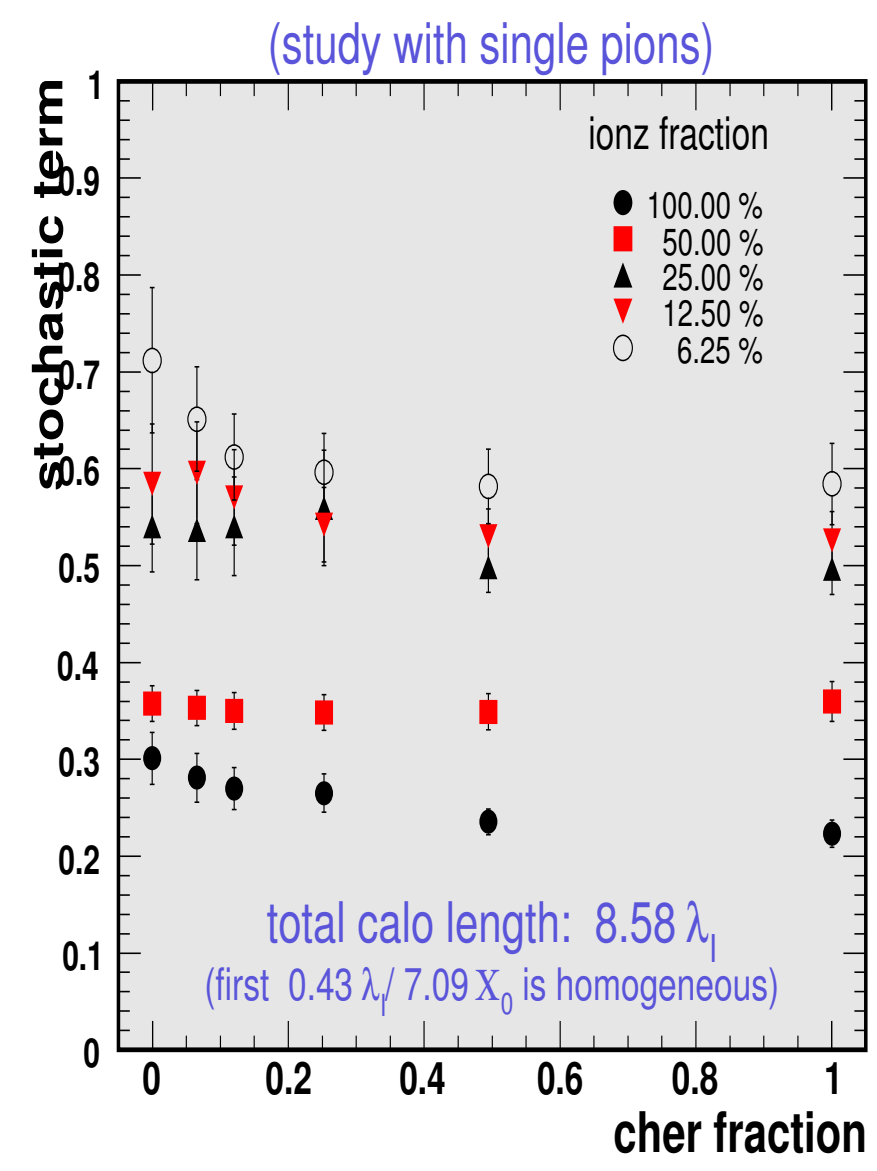

with $7 \mathrm{X}_{0}$ homogeneous part

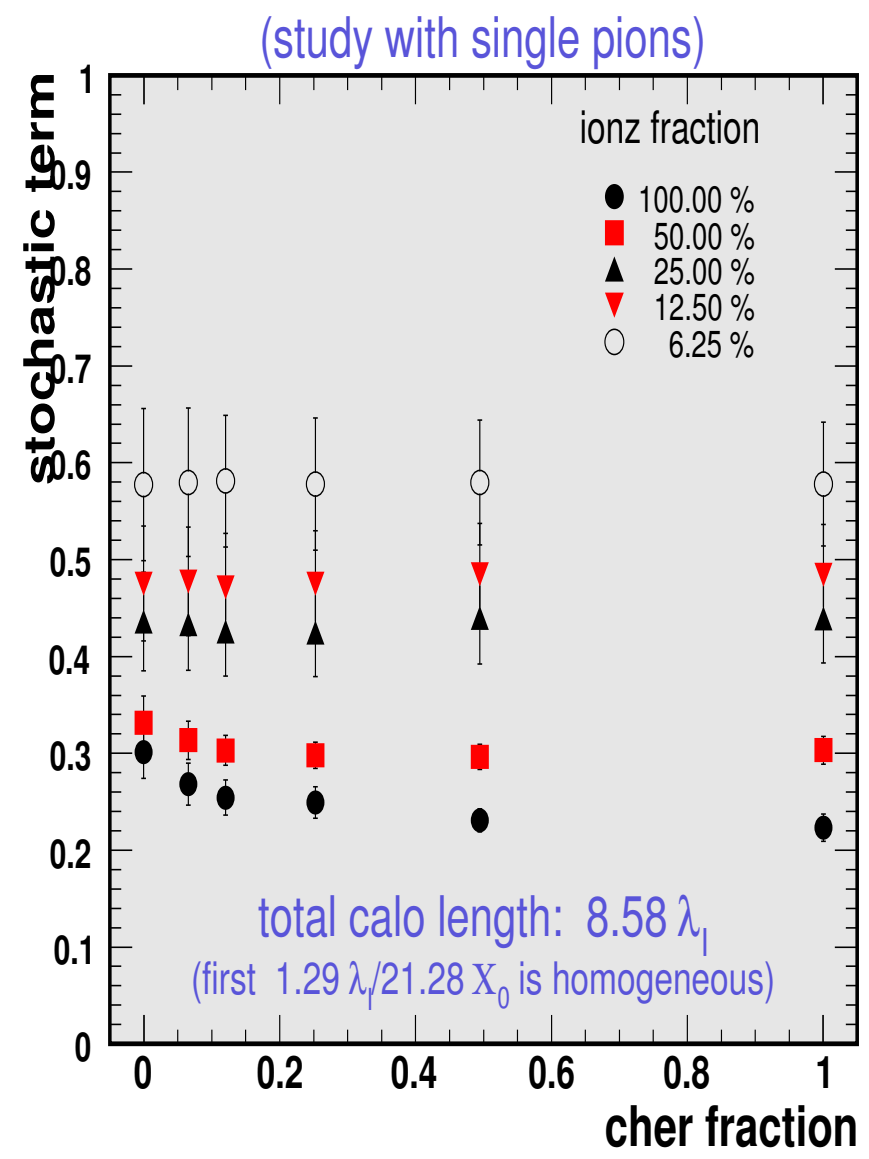

with $21 \mathrm{X}_{0}$ homogeneous part

(homogeneous part+volume of various readout fractions) 


\section{Stochastic term for various configurations}

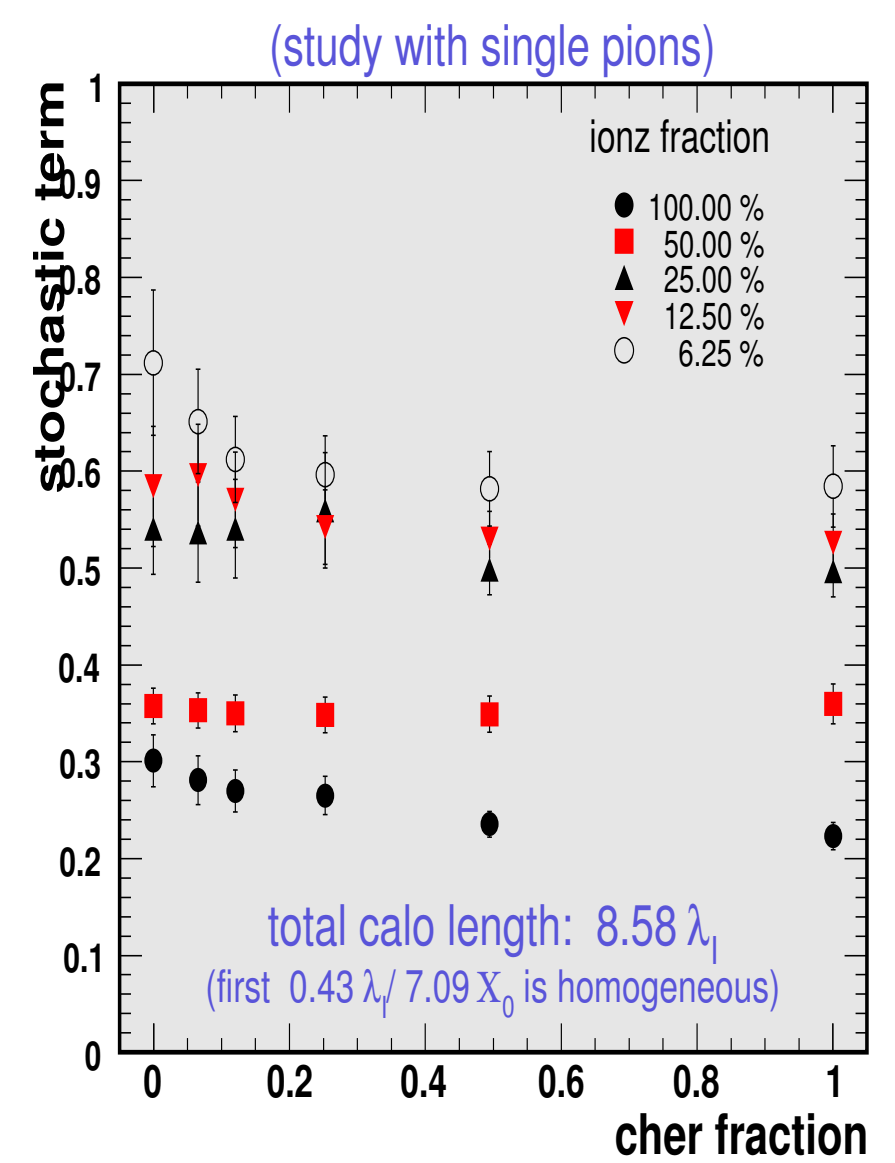

with $7 \mathrm{X}_{0}$ homogeneous part

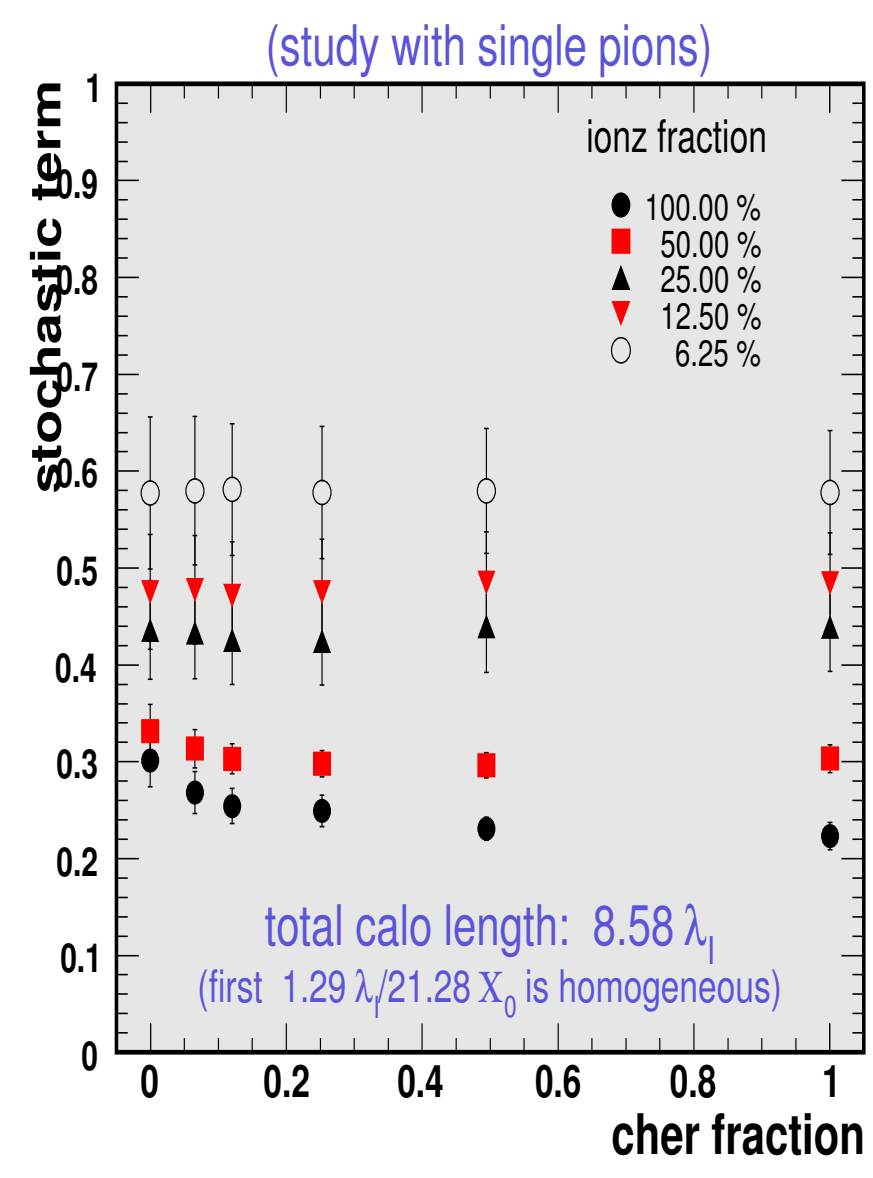

with $21 \mathrm{X}_{0}$ homogeneous part

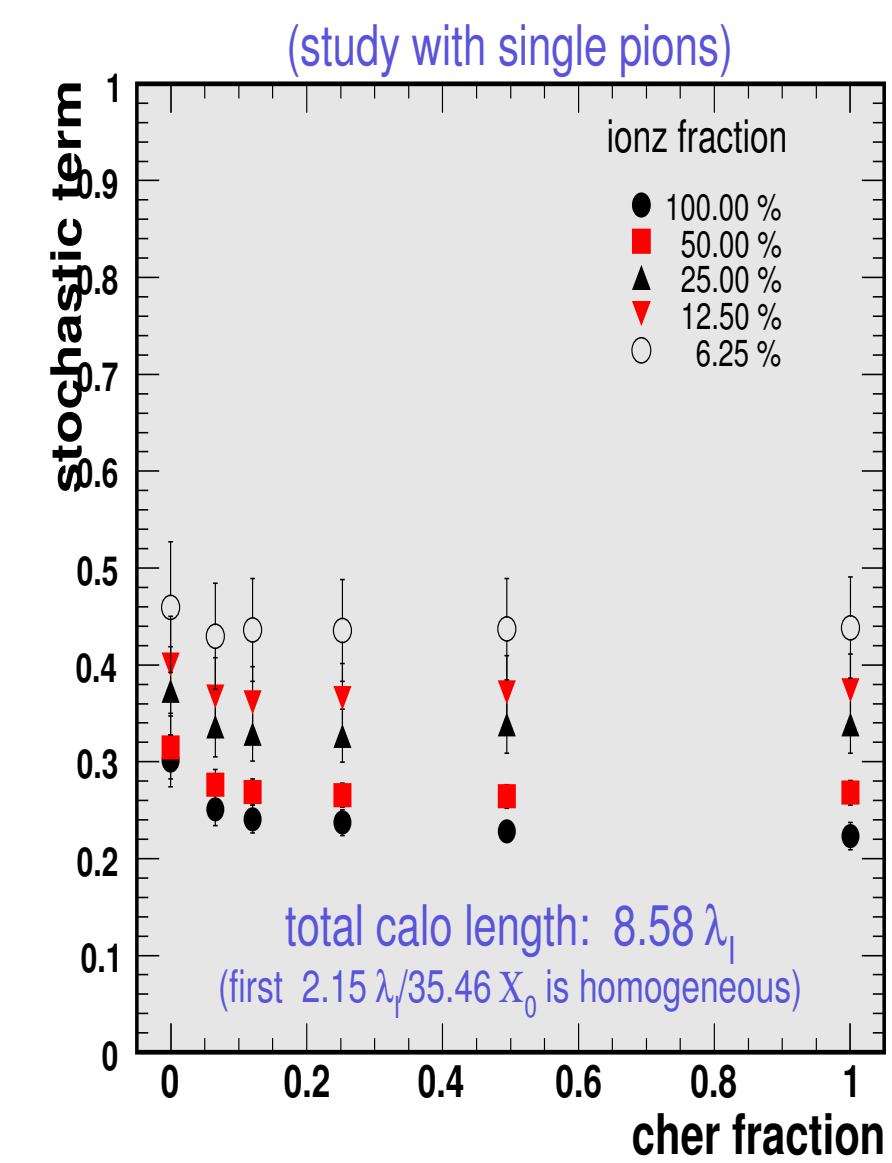

with $35 \mathrm{X}_{0}$ homogeneous part

"homogeneity" improves performance significantly 


\section{Performance trends}

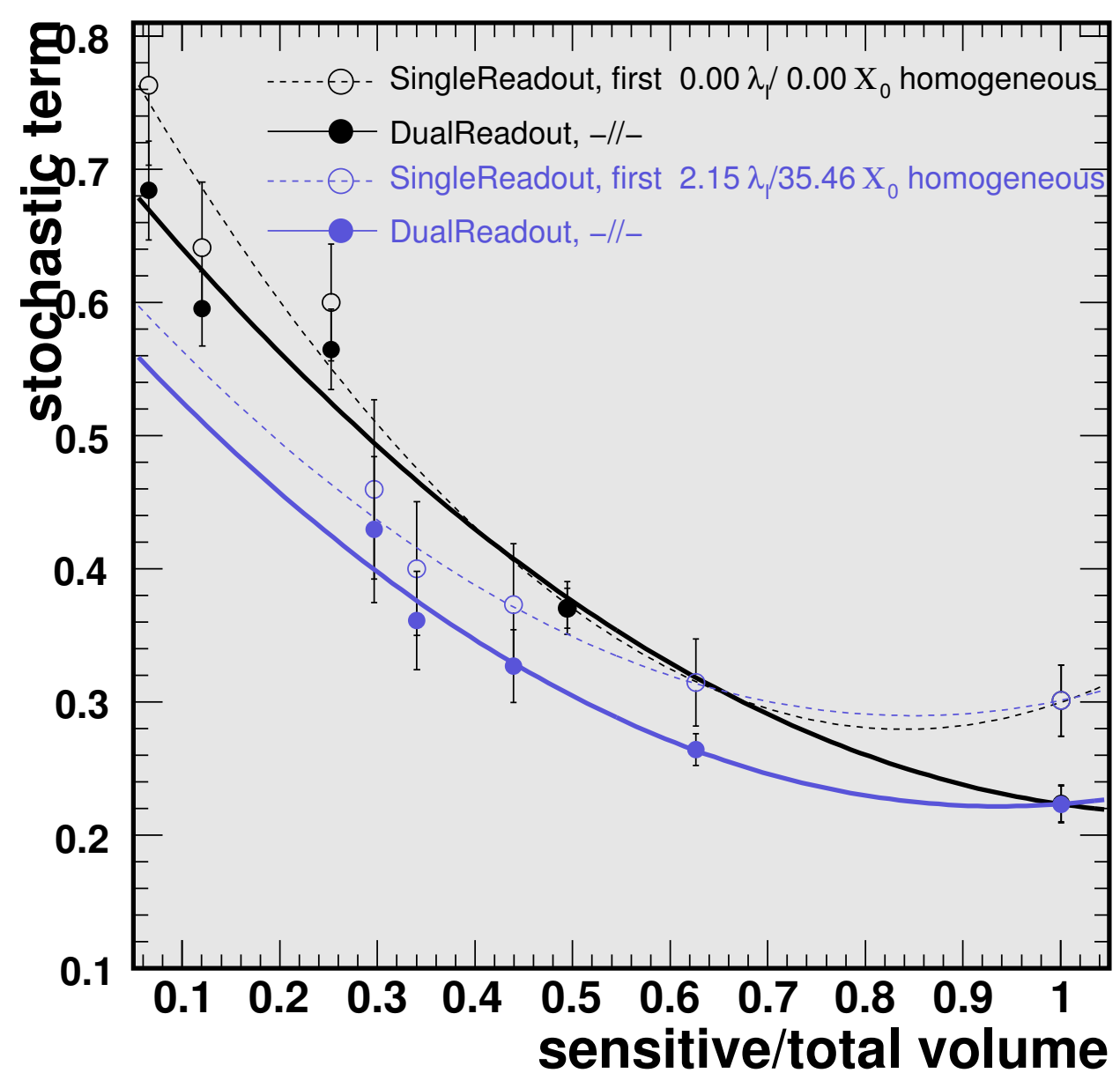

- preliminary trend lines for preliminary performance-cost considerations

- simulated volumes composed of LuAG $\left(\rho=6.73 \mathrm{gr} / \mathrm{cm}^{3}, Z\right.$ Zeff $\left.=62.9, X_{0}=1.41 \mathrm{~cm}, \lambda_{I}=23.3 \mathrm{~cm}\right)$ 


\section{Open questions}

- Design issues and practical questions

: though we are at the very early stage of development of such a concept we always have in mind some design issues that should be studied soon and which need rigorous $\mathbf{R} \& \mathbf{D}$ effort and prototyping

$\triangleright$ material production and cost drivers

$\triangleright$ readout scheme

$\triangleright$ construction

$\triangleright$ scale-up problems

$\triangleright+\ldots$

: can only be answered through the usual phase of prototype development, test and study of 1 permille $\rightarrow 1$ percent $\rightarrow 10$ percent modules of the final detector 


\section{Summary - Outlook}

- metacrystals for calorimetry

: R\&D effort on 3 fronts

$\triangleright$ material development

$\triangleright$ testbeam activities

$\triangleright$ simulation studies

: briefly discussed first results with bundles of crystal fibers exposed to beam and mc parametric scan of an ideal calorimeter

\section{- next steps}

: continue simulation studies and material development

: near-term goal to build a multichannel module (e.g. miniEcal) and expose it to beam 